Supporting information

\title{
Double Layer Capacitance Measurements to Characterize the Water Intrusion into Porous Materials
}

Pradeep Kumar Sow, Zhaowei Lu, Hoda Talebian, Luke Damron, Walter Mérida*

Clean Energy Research Centre, The University of British Columbia, 6250 Applied Science Lane,

Vancouver, BC Canada V6T 1Z4

*Corresponding author: Tel: +1.604.822.4189 Fax: +1.604.822.2403 E-mail:

walter.merida@ubc.ca 


\section{S.1 Intrusion cell components}
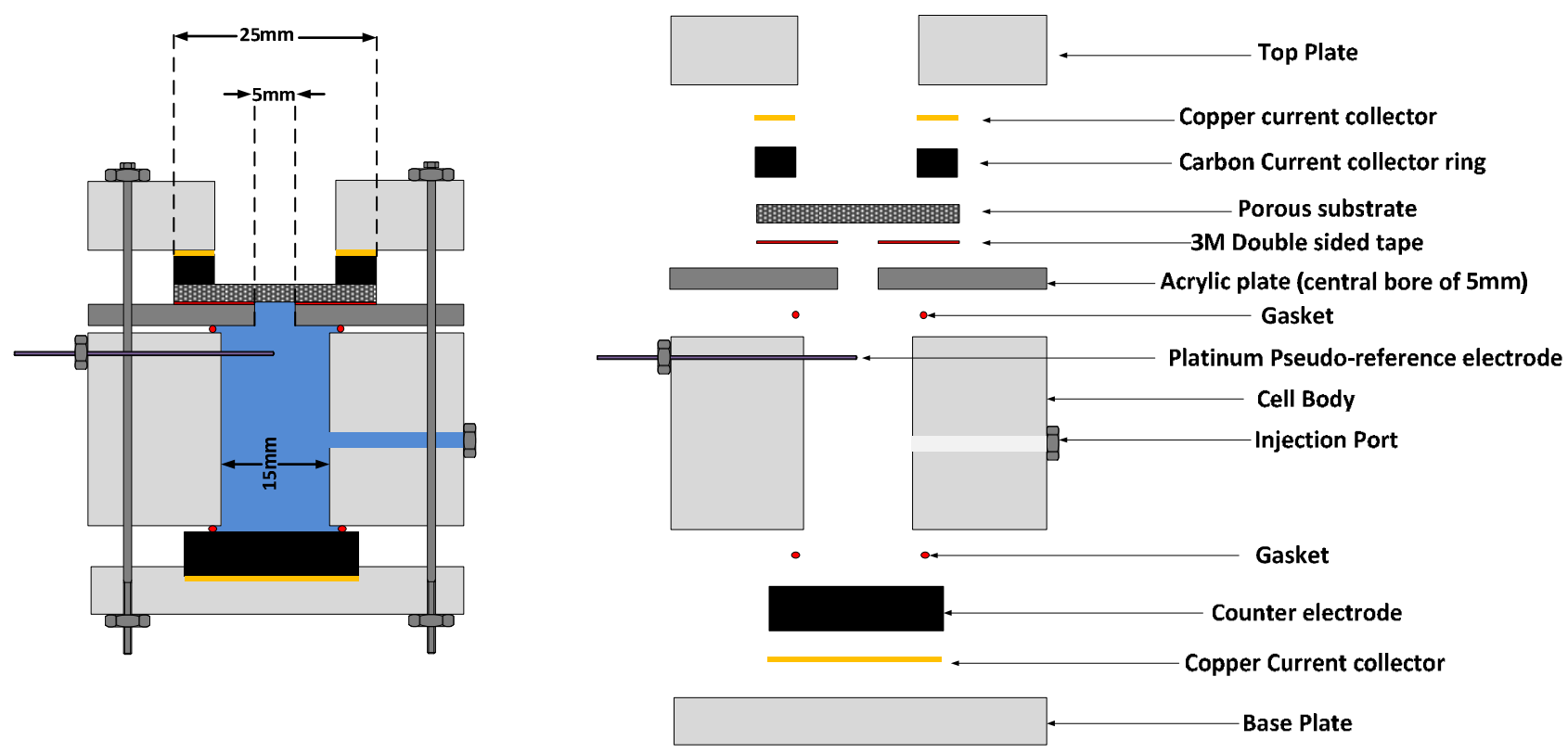

Figure S1: Schematic representation of the intrusion cell components

Figure S1 shows the schematic representation of the intrusion cell used in the present study. The Pt pseudo-reference electrode was placed at a distance of $10 \mathrm{~mm}$ from the sample. In the present configuration, the porous substrate acts as the working electrode in the three electrode system. The samples of diameter $25 \mathrm{~mm}$ were placed on an acrylic plate with a bore diameter of $5 \mathrm{~mm}$ and held in place with a $3 \mathrm{M}$ double sided tape. The bore size on the acrylic plate corresponds to the active area of the substrate through which the intrusion occurs. A ring shaped carbon current collector with an internal diameter of $15 \mathrm{~mm}$ was pressed against the substrate. The inner edge of the current collector ring was at a distance of $5 \mathrm{~mm}$ from the active area to ensure that no fiber breakage occurs near the active area of the substrate through which the intrusion occurs. 


\section{S.2. Proportionality between the double layer capacitance and area}

In the present measurement, five samples of carbon fiber substrate TGP-120 and 10 AA with varying area were prepared. The samples were prepared by covering the substrate with an insulating polyimide tape (Kapton tape, DuPont) while leaving a predetermined uncovered area $\left(\mathrm{A}_{\mathrm{geo}}\right)$ (Figure $\mathrm{S} 1(\mathrm{a})$ ). For the five samples prepared in this work, the uncovered area was varied in the range of $31.7 \mathrm{~mm}^{2}$ to $158.5 \mathrm{~cm}^{2}$. It is to be noted that the area of this uncovered section is significantly higher than the fiber dimensions $(5-15 \mu \mathrm{m})$ as well as the surface structures. Therefore, the distribution of the fiber in the exposed area can be considered homogenous. In order to validate the proportionality between the double layer capacitance $\left(C_{d l}\right)$ and the wetted area $\left(A_{s l}\right)$ for the carbon fiber substrate, independent ex-situ measurements were conducted in a three electrode system as shown in Figure S2 (b). In the experimental measurement, the samples were dipped in the electrolyte solution exposing the uncovered area to the electrolyte solution. The depth $(d)$ to which the sample was immersed in the solution was kept the constant at $12 \mathrm{~mm}$ for all the samples. This arrangement was done to ensure that the hydrostatic pressure due to the liquid remains the same for all samples. The electrolyte used for the measurement was $0.1 \mathrm{M}$ $\mathrm{Na}_{2} \mathrm{SO}_{4}$. A carbon counter electrode and platinum pseudo-reference electrode were used to maintain the consistency with the intrusion experiments.

The capacitance was measured using Cyclic Voltammetry (CV) in the potential range of 0.2 to $0.2 \mathrm{~V}$ w.r.t. Pt Pseudo reference electrode at the scan rate of $200 \mathrm{mV} / \mathrm{s}$. The measurements were conducted for all the samples with varying area exposed to the electrolyte. The capacitance was evaluated from the $\mathrm{CV}$ data using the Eq.2 in the manuscript. The measured capacitance was plotted against the geometric area exposed to the electrolyte $\left(\mathrm{A}_{\mathrm{geo}}\right)$ and the evaluated values are shown in Figure S2 (c) for both TGP-120 and 10AA. The capacitance was found to increase 
linearly with the area as can be observed from the linear fitting of the evaluated data in Figure S2 (c). The above experiment shows that the measured capacitance is proportional to the geometric area $\left(\mathrm{A}_{\text {geo }}\right)$. As the fibers can be assumed homogenously distributed in the exposed area, the wetted area $\left(A_{s l}\right)$ on the surface should be proportional to the exposed geometric area $\left(\mathrm{A}_{\text {geo }}\right)$. Therefore, the evaluated double layer capacitance $\left(C_{d l}\right)$ is proportional to the wetted interfacial area $\left(A_{s l}\right)$ for the materials used in this study.

a)

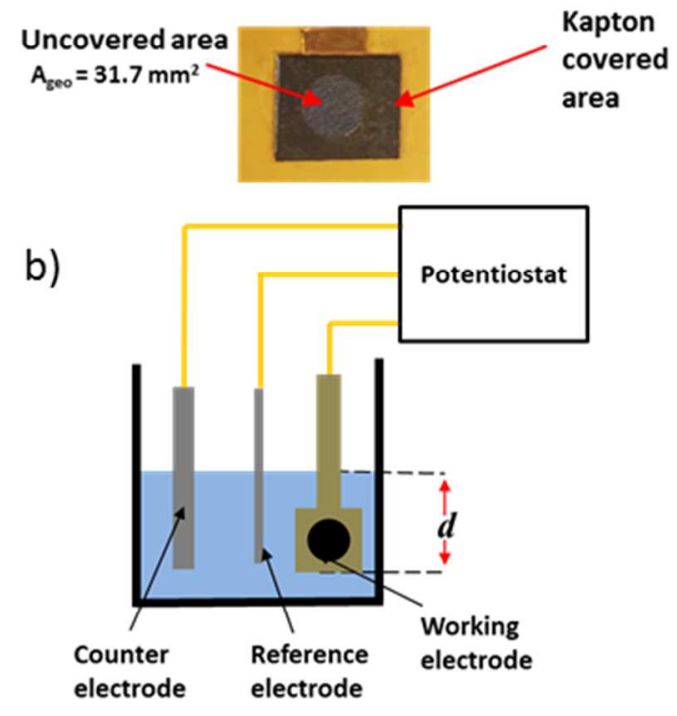

c)

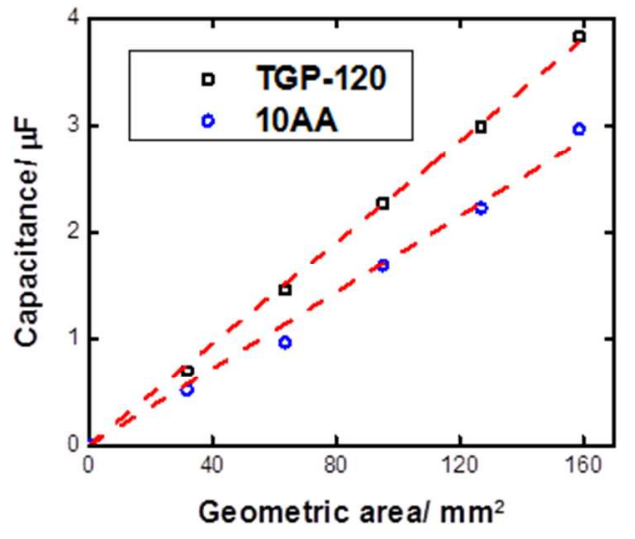

Figure S2: a) Photograph of a polyimide tape covered substrate with an uncovered area $\left(\mathrm{A}_{\mathrm{geo}}\right)$ of $31.7 \mathrm{~mm}^{2}$. b) Schematic representation of the three electrode experimental setup. Note that the depth $(d)$ to which the sample has been immersed in the electrolyte was kept constant at $12 \mathrm{~mm}$ for all the samples. c) Figure showing the plot of the capacitance evaluated from the CV curve and the geometric area exposed to the electrolyte. Linear fitting shows a proportional relationship between area and capacitance. 